\title{
The Equality of Fractal Dimension and Uncertainty Dimension for Certain Dynamical Systems ${ }^{\star}$
}

\author{
Helena E. Nusse ${ }^{1,2}$ and James A. Yorke ${ }^{1,3}$ \\ ${ }^{1}$ Institute for Physical Science and Technology, University of Maryland, College Park, \\ MD 20742, USA \\ ${ }^{2}$ Rijksuniversiteit Groningen, Fac. Economische Wetenschappen, WSN-gebouw, Postbus 800, \\ NL-9700 AV Groningen, The Netherlands \\ 3 Department of Mathematics, University of Maryland, College Park, MD 20742, USA
}

Received March 28, 1991

\begin{abstract}
MGOY] introduced the uncertainty dimension as a quantative measure for final state sensitivity in a system. In [MGOY] and [P] it was conjectured that the box-counting dimension equals the uncertainty dimension for basin boundaries in typical dynamical systems. In this paper our main result is that the box-counting dimension, the uncertainty dimension and the Hausdorff dimension are all equal for the basin boundaries of one and two dimensional systems, which are uniformly hyperbolic on their basin boundary. When the box-counting dimension of the basin boundary is large, that is, near the dimension of the phase space, this result implies that even a large decrease in the uncertainty of the position of the initial condition yields only a relatively small decrease in the uncertainty of which basin that initial point is in.
\end{abstract}

\section{Introduction}

Nonlinear dynamical systems often have more than one attractor, and it is of fundamental importance to be able to determine which attractor a specified initial condition goes to. We are interested in the basin boundary, that is, common boundary between the basins of the attractors. For example, for suitably chosen parameter values, the Hénon map has a fractal basin boundary between the points whose orbits go to $\infty$ (infinity is an attractor) and the points whose trajectories remain bounded and go to the chaotic attractor. When the basin boundary is fractal, it follows that there is a non-attracting, compact, chaotic, invariant set in the basin boundary. Examples with fractal basin boundaries are common and occur for example in the forced damped pendulum and the forced Duffing equation.

The fact that a basin boundary is fractal does have important practical consequences. In particular, for the purposes of determining which attractor eventually

* Research in part supported by AFOSR and by the Department of Energy (Scientific Computing Staff Office of Energy Research) 
captures a given orbit, the arbitrarily fine-scaled structure of fractal basin boundaries implies considerable sensitivity to small errors in initial conditions.

If we assume that in a physical situation initial points cannot be located more precisely than some $\varepsilon>0$, then we cannot determine which basin a point is in if it is within $\varepsilon$ of the basin boundary. Such points are called $\varepsilon$-uncertain. It is easy to check that the Lebesgue measure of the set of $\varepsilon$-uncertain points (in a bounded region of interest) scales like $\varepsilon^{m-d}$, where $m$ is the dimension of the phase space and $d$ is the box-counting dimension of the basin boundary. Notice that when $m-d$ is near 0 , a large decrease in $\varepsilon$ results in a small decrease in $\varepsilon^{m-d}$. Cases where $m-d<0.1$ are common. This is discussed in [GMOY] and [MGOY], where it is also shown that the basin boundary dimension provides a quantitative measure of sensitivity.

Let $M$ denote either a compact, smooth $m$-dimensional manifold without boundary, where $m \geqq 2$, or an interval on the real line. Let $F$ be a $C^{3}$-diffeomorphism from $M$ to itself when $m \geqq 2$, and let $f$ be a $C^{1+\alpha}$-map from $M$ to itself when $m=1$ (that is, there exist constants $K>0$ and $\alpha>0$ such that $\left|f^{\prime}(x)-f^{\prime}(y)\right|$ $\leqq K \cdot|x-y|^{\alpha}$ for all $x, y$ in $M$, where $f^{\prime}$ is the derivative of $f$ ). For convenience, in this paper we assume throughout that for any one-dimensional map $f$ there exists a compact interval $I$ in the interior of $M$ such that for every $x \in M$ there is an integer $n \geqq 0$ for which the iterate $f^{n}(x)$ of $x$ is in $I$. Other cases are analyzed similarly; see [Nu1]. For $x, y$ in $M$ we write $\rho(x, y)$ for the distance between $x$ and $y$. From now on, we write $g$ to denote either $F$ or $f$.

A set $S \subset M$ is called positively $g$-invariant if $g(S) \subset S$, and is called $g$-invariant if $g(S)=S$. For a closed set $S \subset M$ and $x \in M$, write $\rho(x, S)=\min \{\rho(x, y): y \in S\}$. An attractor $A$ is a $g$-invariant, compact set in $M$ such that (1) there exists an open neighborhood $U$ of $A$ such that for each $x \in U$ the distance $\rho\left(g^{n}(x), A\right) \rightarrow 0$ when $n \rightarrow \infty$; and (2) there is a point $x \in A$ such that the closure of the trajectory $\left\{g^{n}(x)\right\}_{n \geqq 0}$ equals $A$. For an attractor $A$ we say, the domain of attraction of $A$ is the set of points $x$ in $M$ for which $\rho\left(g^{n}(x), A\right) \rightarrow 0$ as $n \rightarrow \infty$. In the literature, for an attractor $A$ the notion "basin of $A$ " is often equivalent with the notion "domain of attraction of $A$ ". On the other hand, in other studies of dynamical systems, the notion "basin of $A$ " is defined as the region in $M$ that is the interior of the closure of the domain of attraction of $A$. Therefore, for an attractor $A$ we define $\operatorname{basin}\{A\}$ to be the interior of the closure of the domain of attraction of $A$. We would like to emphasize that $\operatorname{basin}\{A\}$ may include $g$-invariant sets that are not in the domain of attraction of $A$; that is, the trajectories of some points in basin $\{A\}$ will not converge to the attractor $A$. For an example of this phenomenon, see the Forced Pendulum Example in [NY1].

The basin boundary is the set of all points $x \in M$ for which each open neighborhood has a nonempty intersection with at least two different domains of attraction, see [GOY]. In other words, the basin boundary is the set of all points $x \in M$ for which there exist two attractors, say $A_{1}$ and $A_{2}$, such that $x$ is on the boundary of both basin $\left\{A_{1}\right\}$ and basin $\left\{A_{2}\right\}$.

Throughout this paper, we assume:

(A1) there exist finitely many, but at least two, attractors, say $A_{1}, A_{2}, \ldots, A_{q}$; and

(A2) for each point $x \in M$, there exists an integer $k, 1 \leqq k \leqq q$, such that either $x \in \operatorname{basin}\left\{A_{k}\right\}$ or $x$ is on the boundary of basin $\left\{A_{k}\right\}$. 
We may say the basin boundary is fractal if its dimension plus one is not equal to the dimension of the phase space. The "dimension" of this basin boundary can be any of several concepts.

We define a region to be an open, bounded set in $M$. The structure of the basin boundary in a region depends on the long-term behavior of trajectories that start on the basin boundary in this region. Basin boundaries are invariant sets and trajectories starting on the basin boundary will asymptote to some set that is usually not the entire basin boundary. This asymptotic set may depend on the initial point. A principal idea of the paper [GNOY] is: (a) if trajectories on the basin boundary in different regions have the same asymptotic behavior, then the dimension of the basin boundary is the same in these different regions, and (b) if in two different regions, the trajectories on the basin boundary have different asymptotic behavior and the basin boundary is fractal, then the probability is zero that the dimension of the basin boundary in the two regions is the same.

Let BB denote the basin boundary, and let $S$ be a region that intersects the basin boundary. We write $\operatorname{dim}(S \cap \mathrm{BB})$ for the dimension (whichever concept of dimension is under discussion) of the part of the basin boundary which lies in $S$. The dimension $\operatorname{dim}(S \cap \mathrm{BB})$ can conceivably take on different values depending on $S$. The question of how many such values are possible has been discussed in [GNOY] and it is proved to be finite for certain one and two dimensional hyperbolic systems. A natural question which arises is then whether different concepts of dimension yield the same values for any given region. In [FOY] it is conjectured that the box-counting dimension equals the Hausdorff dimension for basin boundaries of typical dynamical systems. Since the box-counting dimension is often much easier to estimate than the Hausdorff dimension, it is worthwhile to know to which extent these two notions of dimension are equal.

Consider an initial condition $x$ that is on the basin boundary and evolve it forward in time, and let $L^{+}(x)$ be the set of limit points of the trajectory $\left\{g^{n}(x)\right\}_{n \geqq 0}$ of the point $x$. Let $L^{+}(\mathrm{BB})$ be the union of the limit points of all trajectories that start on the basin boundary (that is, $L^{+}(\mathrm{BB})=\bigcup\left\{L^{+}(x): x \in \mathrm{BB}\right\}$ ). For uniformly hyperbolic systems there exists a finite set $S$ of initial points such that $L^{+}(\mathrm{BB})$ is the disjoint union of "basic sets" $L^{+}(x)$ where $x \in S$. Assume that we have two points, $x_{a}$ and $x_{b}$, on the basin boundary for which $L^{+}\left(x_{a}\right)$ and $L^{+}\left(x_{b}\right)$ are contained in the same basic set. Then, from [GNOY] it is known that if we take a sufficiently small neighborhood $S_{a}$ of $x_{a}$ and a sufficiently small neighborhood $S_{b}$ of $x_{b}$, the dimension of the basin boundary in these two neighborhoods is the same, that is, $\operatorname{dim}\left(S_{a} \cap \mathrm{BB}\right)=\operatorname{dim}\left(S_{b} \cap \mathrm{BB}\right)$, where $\operatorname{dim}$ denotes either the box-counting or Hausdorff or uncertainty dimension.

In this paper we show that $d_{b}(S \cap \mathrm{BB})=d_{u}(S \cap \mathrm{BB})=d_{H}(S \cap \mathrm{BB})$, where $S$ is any region and where $d_{b}, d_{u}$, respectively, $d_{H}$ denote the box-counting dimension, the uncertainty dimension, respectively the Hausdorff dimension (see Sect. 2 for the definitions).

Our results include the class of one dimensional maps and the "linear" horseshoe example presented by Pelikan [P]. In the current literature the notion "fractal dimension" means in almost all cases "box-counting dimension." However, Eggleston [E] explicitly distinguished Hausdorff dimension and fractal dimension, and the commonly used notion "Hausdorff dimension" defined below is "fractal dimension" in [E]. Takens [T] showed that the Hausdorff dimension and the boxcounting dimension of dynamically defined Cantor sets of hyperbolic diffeomorphisms are equal. Some basic sets on the basin boundary are attractors relative to 
the basin boundary, that is, the trajectories of points on the basin boundary near such a basic set are attracted to it. It is however possible for the basin boundary to contain basic sets that are not attractors relative to the boundary, and such cases can be structurally stable. While the Takens' result does not cover such cases, our results do allow such complications.

The organization of the paper is as follows. In Sect. 2 the definitions concerning hyperbolicity and dimensions are stated. The results are precisely stated in Sect. 3. Finally, in Sect. 4 the proofs of the results are presented.

\section{Definitions}

2A. Hyperbolicity. Let the manifold $M$ and the map $g$ be as in the introduction. Recall that $g$ is a $C^{1+\alpha}$ map when $M$ is an interval and $g$ is a $C^{3}$ diffeomorphism when $m=\operatorname{dim}(M) \geqq 2$.

A point $x$ in $M$ is called a nonwandering point for $g$, if for each open neighborhood $U$ of $x$ there exists a positive integer $n$ such that $g^{n}(U)$ and $U$ intersect. The set of all nonwandering poins for $g$ is called the nonwandering set of $g$, and it is denoted by $\Omega(g)$.

For $m \geqq 2$, a subset $\Lambda$ of $M$ is hyperbolic if it is compact, $F$-invariant, and the tangent bundle $T_{A} M$ splits into $d F$-invariant subbundles $E^{s}$ and $E^{u}$ on which $d F$ is uniformly contracting and uniformly expanding respectively. A hyperbolic set $\Lambda$ is called saddle-hyperbolic if $\operatorname{dim} E^{s} \geqq 1$ and $\operatorname{dim} E^{u} \geqq 1$. The map $F$ is called an Axiom $A$ diffeomorphism if the nonwandering set $\Omega(F)$ is hyperbolic and the periodic points of $F$ are dense in $\Omega(F)$. If $F$ is an Axiom $A$ diffeomorphism, then by Smale's "Spectral Decomposition Theorem", the set $\Omega(F)$ can be decomposed uniquely into a finite collection of disjoint, compact, invariant subsets having the property that if $\Gamma$ is any of these subsets, then (1) $F$ has a dense orbit on $\Gamma$ and (2) $\Gamma$ is the maximal invariant set in a neighborhood of $\Gamma$; subsets of $\Omega(F)$ that satisfy (1) and (2), are called basic sets (see e.g. [GH] for a discussion of uniformly hyperbolic systems). If $m=2$ and if $\Omega(F)$ is saddle-hyperbolic, then by a result due to Newhouse and Palis [NP] we know that the periodic points of $F$ are dense in $\Omega(F)$. This implies that if $\Omega(F)$ is hyperbolic and $m=2$, then $F$ is an Axiom $A$ diffeomorpism.

For $m=1$, a subset $\Lambda$ of $M$ is hyperbolic if $\Lambda$ can be decomposed into positively $f$-invariant, compact sets $\Lambda_{a}$ and $\Lambda_{e}$ on which $f^{\prime}$ is uniformly contracting and expanding respectively. In [Nu2], the map $f$ is called an Axiom $A$ map if $\Omega(f)$ is hyperbolic; the periodic points are automatically dense in $\Omega(f)$. A point $x$ in $M$ is a critical point if $f^{\prime}(x)=0$. We say that the $\operatorname{map} f$ is non-critical if for each critical point $c$ of $f$, the intersection of the limit set $L^{+}(c)$ and $\Omega_{e}(f)$ is empty. Note that $L^{+}(c)$ is a subset of $\Omega(f)$. If $f$ is an Axiom $A$ map, then $(1) \Omega(f)$ can uniquely be decomposed into finitely many isolated, compact, positively $f$-invariant sets, called basic sets, and $f$ has a dense orbit on each of these basic sets, and (2) the periodic points of $f$ are dense in $\Omega(f)$; see [Nu2] for details.

We say that $g$ is non-critical when $g=f$ if $f$ is a non-critical map, and when $g=F$ we assume $F$ is automatically non-critical since the Jacobian matrix of $F$ is non-singular.

2B. Dimension Definitions for Basin Boundaries. We will recall some definitions of different dimensions and some of their properties. Throughout this paper, we write 
BB for the basin boundary, and we assume $S$ to be a region that intersects the basin boundary. The pointwise dimensions of the basin boundary $\mathrm{BB}$ at $x$ is defined as

$$
\operatorname{dim} \mathrm{BB}(x)=\lim _{\varepsilon \rightarrow 0} \operatorname{dim}(B(x ; \varepsilon) \cap \mathrm{BB}),
$$

where $B(x ; \varepsilon)$ denotes a ball with radius $\varepsilon$ centered at $x$. Since the basin boundary is assumed to be compact, we say the dimension of the basin boundary $\operatorname{dim}(\mathrm{BB})$ is defined by the maximum value of the pointwise dimensions $\operatorname{dim} B B(x)$ for $x$ in the basin boundary $\mathrm{BB}$, that is, $\operatorname{dim}(\mathrm{BB})=\max \{\operatorname{dim} \mathrm{BB}(x): x \in \mathrm{BB}\}$. Throughout this paper, we assume that the $\operatorname{limit}_{\lim _{\varepsilon \rightarrow 0}} \ln N(\varepsilon, S) / \ln (1 / \varepsilon)$ exists. When the limit does not exist, we say the box-counting dimension does not exist. We prefer to avoid non-generic situations. The box-counting (or capacity) dimension of $\mathrm{BB}$ in $S$ is defined as

$$
d_{b}(S \cap \mathrm{BB})=\lim _{\varepsilon \rightarrow 0} \ln N(\varepsilon, S) / \ln (1 / \varepsilon)
$$

where $N(\varepsilon, S)$ denotes the minimum of $m$-dimensional cubes in a grid of edge length $\varepsilon$ needed to cover that part of the basin boundary which lies in $S$, and $m$ is the dimension of the phase space $M$. It can be shown, see [MGOY], that $d_{b}(S \cap \mathrm{BB})$ can also be defined as

$$
d_{b}(S \cap \mathrm{BB})=m-\lim _{\varepsilon \rightarrow 0} \ln \varphi(\varepsilon, S) / \ln \varepsilon,
$$

where $\varphi(\varepsilon, S)$ is the fraction of the phase space volume lying in $S$ which is within $\varepsilon$ of $S \cap$ BB. From a certain practical point of view, see [GMOY, MGOY], this latter definition is more useful.

We now consider another definition of a dimension of the basin boundary. We assume that $S \cap \mathrm{BB}$ is not empty. Say we randomly pick an initial condition $x$ with uniform probability in $S$. Then we pick another initial condition $y$ in $S$ randomly in the ball $|x-y| \leqq \varepsilon$. Let $p(\varepsilon, S)$ be the probability that $x$ and $y$ are in different basins. (We can think of $p(\varepsilon, S)$ as the probability that an error will be made in determining the basin of an initial condition if the initial condition has uncertainty of size $\varepsilon$.) We define the uncertainty dimension of BB in $S$ as

$$
d_{u}(S \cap \mathrm{BB})=m-\lim _{\varepsilon \rightarrow 0} \ln p(\varepsilon, S) / \ln \varepsilon
$$

When $\mathrm{BB}$ is a smooth surface, $d_{u}(S \cap \mathrm{BB})=m-1$, and $p(\varepsilon, S) \propto \varepsilon$. However, for fractal basin boundaries, we can have $p(\varepsilon, S) \propto \varepsilon^{\alpha}$ with $\alpha=m-d_{u}(S \cap \mathrm{BB})<1$, indicating enhanced sensitivity to small uncertainty in initial conditions. For example, one numerically obtains $m-d_{u}(\mathrm{BB}) \approx 0.275$ for an example involving a periodically forced pendulum in [GNOY]. In this case a decrease of the initial condition uncertainty $\varepsilon$ by a factor of 10 leads to only a relative small decrease in the final state uncertainty $p(\varepsilon, S)$, since $p$ decreases by a factor of about $10^{0.275} \approx 1.9$. Thus, in practical terms, it may be essentially impossible to significantly reduce the final state uncertainty in some experimental situations. In [MGOY] and [P] it has been conjectured that $d_{b}(\mathrm{BB})=d_{u}(\mathrm{BB})$ for basin boundaries in typical dynamical systems. 
Now we define the Hausdorff dimensions for a compact set $G$ in the plane. For each $a>0$, we consider

$$
h(a)=\lim _{\varepsilon \rightarrow 0} \inf _{G} \sum_{A_{i} \in G}\left[\operatorname{diam}\left(A_{i}\right)\right]^{a},
$$

where for each $\varepsilon>0$ the inf is taken over all possible countable covers of $G$ whose elements $A_{i}$ have diameter $\operatorname{diam}\left(A_{i}\right)$ less than $\varepsilon$. The Hausdorff dimension $d_{H}(G)$ of $G$ is defined as

$$
d_{H}(G)=\inf \{a: h(a)=0\} .
$$

In general, one has $d_{H}(G) \leqq d_{b}(G)$.

\section{Results}

Throughout, we assume that either $m=1$ or $m=2$. Let the manifold $M$, the map $g$, and the basin boundary BB as above. In [GNOY] we showed that for one dimensional non-critical Axiom $A$ maps and two dimensional Axiom A diffeomorphisms, the number of basic sets in the basin boundary is an upper bound on the number of different values of the basin boundary for each of the notions of dimension discussed in this paper. That is, the number of values of $\operatorname{dim}(S \cap \mathrm{BB})$ considering all open sets $S$ which intersect BB is bounded from above by the number of basic sets in BB.

The crucial assumptions in obtaining the main result (stated below) are encapsulated in the following definitions. We define the map $g$ to be hyperbolic on the basin boundary if $g$ is non-critical and $\mathrm{BB} \cap \Omega(g)$ is a non-empty hyperbolic set. We would like to mention that the uniformly hyperbolic systems studied in [GNOY] satisfy these conditions.

Theorem. Let g: $M \rightarrow M$ satisfy the following conditions:

(A1) there exist finitely many, but at least two, attractors, say $A_{1}, A_{2}, \ldots, A_{q}$;

(A2) for each point $x \in M$, there exists an integer $k, 1 \leqq k \leqq q$, such that either $x \in$ basin $\left\{A_{k}\right\}$ or $x$ is on the boundary of basin $\left\{A_{k}\right\}$;

(A3) $g$ is hyperbolic on the basin boundary $\mathrm{BB}$.

Then, for each region $S$ that intersects the basin boundary, the box-counting dimension $d_{b}(S \cap \mathrm{BB})$, the Hausdorff dimension $d_{H}(S \cap \mathrm{BB})$ and the uncertainty dimension $d_{u}(S \cap \mathrm{BB})$ of the intersection of $S$ with the basin boundary BB are all equal.

Corollary. Let $g$ be as in the theorem. Then taking $S=M$, the box-counting dimension $d_{b}(\mathrm{BB})$, the Hausdorff dimension $d_{H}(\mathrm{BB})$, and the uncertainty dimension $d_{u}(\mathrm{BB})$ of the basin boundary $\mathrm{BB}$ are all equal.

The basin boundaries of the one dimensional maps considered so far are boundaries of basins of periodic orbits. Our proofs easily extend to a more general class of attractors. In the literature, several definitions of "attractor" appear, see e.g. Milnor [Mi]. We say, a Milnor-attractor $A$ is a positively $f$-invariant, compact set in $M$ such that (1) for each open neighborhood $U$ of $A$ there exists a set $W$ in $U$ with positive Lebesgue measure, such that for each $x$ in $W$ the distance $\rho\left(g^{n}(x), A\right) \rightarrow 0$ 
when $n \rightarrow \infty$; and (2) there is a point $x \in A$ such that the closure of $\left\{g^{n}(x)\right\}_{n \geqq 0}$ equals $A$. For a Milnor-attractor $A$ we say, the domain of attraction of $A$ is the set of all points $x$ in $M$ for which $\rho\left(g^{n}(x), A\right) \rightarrow 0$ as $n \rightarrow \infty$. Note that the domain of attraction of a Milnor-attractor is not necessarily open. Similarly as for an attractor, we define for a Milnor-attractor $A$ basin $\{A\}$ to be the interior of the closure of the domain of attraction of $A$. For the quadratic map $f_{a}(x)=\operatorname{ax}(1-x)$, note that basin $\{A\}$ of a Milnor-attractor $A$ equals the open interval $(0,1)$, where $0<a<4$. In order to formulate a more general result, we consider finitely many, disjoint, positively $f$-invariant, open sets each having its own Milnor-attractor. Notice that $A$ may be a one-sided or two-sided attractive periodic orbit (simple attractor), homeomorphic to a Cantor set (strange attractor), or consist of finitely many nontrivial compact intervals (chaotic attractor).

Proposition. Assume that for some integer $n \geqq 2$, there exist $n$ Milnor-attractors $A_{k}, 1 \leqq k \leqq n$, in $M$ such that (writing $O_{k}=\operatorname{basin}\left\{A_{k}\right\}$ ),

(1) the union of these open sets $\bigcup_{k=1}^{n} O_{k}$ is dense in $M$;

(2) the $O_{k}$ 's are disjoint open sets;

(3) the map $f$ is hyperbolic on the basin boundary.

Then, for each open interval $S$,

$$
d_{b}(S \cap \mathrm{BB})=d_{H}(S \cap \mathrm{BB})=d_{u}(S \cap \mathrm{BB}) .
$$

Remark 1. If $f$ is as in the proposition, then the box-counting dimension, the Hausdorff dimension, and the uncertainty dimension of the basin boundary are all equal.

Remark 2. Let $f$ be as in the proposition. We have not excluded the possibility that $f$ may have a one-sided stable periodic orbit $P$, but in such cases $P$ cannot be on the basin boundary (because of assumption (3)).

Remark 3. If $f: M \rightarrow M$ satisfies the conditions: (1) $f$ has a nonpositive Schwarzian derivative; (2) every bounded attractor is a periodic orbit; (3) $f$ has finitely many critical points and for each critical point $c$ there exists an attractor $A$ such that $\rho\left(f^{n}(c), A\right) \rightarrow 0$ as $n \rightarrow \infty$; then $f$ is a non-critical Axiom $A$ map (see [Nu2]). Therefore, the results above hold for these maps too.

Example. The purpose of this example is to show that the condition that $g$ is hyperbolic on the basic boundary is essential. The objective in [LY] is to show that piecewise $C^{1}$ expanding maps on the unit interval have absolutely continuous invariant measures. In [LY] it is shown that the map $\gamma$ defined by $\gamma(x)=x /(1-x)$ for $0 \leqq x \leqq 0.5$ and $\gamma(x)=2-2 x$ for $0.5 \leqq x \leqq 1$, which violates the assumption $\left|\gamma^{\prime}(x)\right|>1$ only at $x=0$, does not have an absolutely continuous invariant measure.

Motivated by this example, we consider a map $f$ (as shown in Fig. 1) from the open interval $\left(-\frac{1}{3}, \frac{3}{2}\right)$ to itself such that (writing $J$ for the open interval $\left(\frac{7}{20}, \frac{3}{2}\right)$ ), (1) $f(y)=y /(1-y)$ for $-\frac{1}{3}<y<\frac{7}{20}$; (2) $f$ has 3 fixed point attractors in $J$; (3) the intersection of the basin boundary of $f$ with $J$ consists of 3 points, namely the repelling fixed points 0.5 and 1 , and the point $\xi$ that is mapped into the fixed point 0.5 , that is, $f(\xi)=0.5$; (4) the Schwarzian derivative of $f$ is nonpositive.

The basin boundary of $f$ consists of the points $0, \xi$, and all the points $1 / n$ where $n$ is any positive integer. Notice $f(1 / n+1)=1 / n$ for $n \geqq 2$. It is easily verified that 


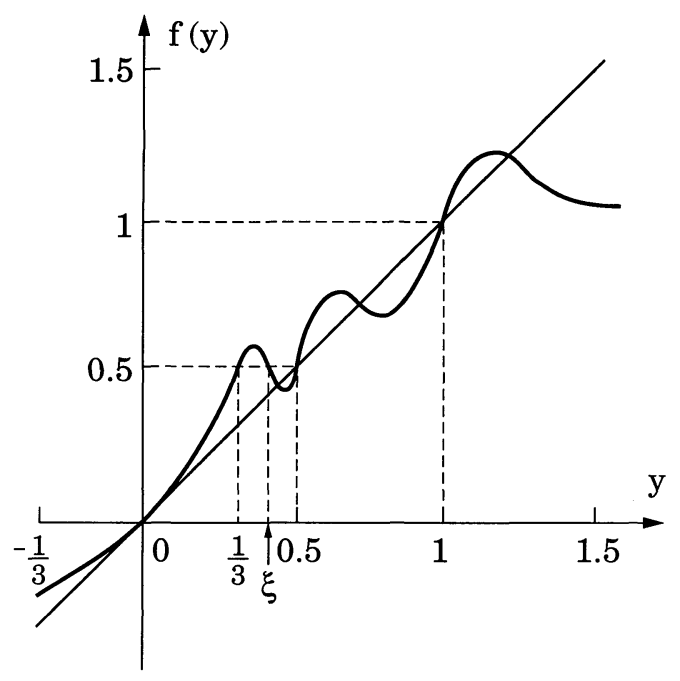

Fig. 1.

the Hausdorff dimension and the box-counting dimension of the basin boundary of $f$ differ. In particular, one can verify that the box-counting dimension of $\{1 / n\}_{n \geqq 2}$ is 0.5 . Hence, the box-counting dimension of the basin boundary is at least 0.5 , while the Hausdorff dimension of the basin boundary is 0 .

We would like to mention that the idea of looking for a map whose basin boundary is the set $1 / 2,1 / 3,1 / 4, \ldots, 1 / n, \ldots$ and 0 , was suggested by Brian Hunt.

\section{Proofs of the Results}

Let the manifold $M$, the distance $\rho$ on $M$, and the map $g$ be as in the introduction, and assume that the dimension of $M$ is either one or two. Assume that (1) $g$ has finitely many attractors (defined as in the introduction), say $A_{1}, \ldots, A_{q}$, where $q \geqq 2$; (2) for each $x \in M$ there exists an integer $k$ such that either $x \in$ basin $\left\{A_{k}\right\}$ or $x$ is on the boundary of basin $\left\{A_{k}\right\}, 1 \leqq k \leqq q$; and (3) $g$ is hyperbolic on the basin boundary. We write BB for the basin boundary, and we assume that $S$ is a region that intersects BB.

4-1. Preliminaries. Recall that for $z \in \Omega(g)$ the stable set $W^{s}(z)$ of $z$ is the set of points $x$ for which $\rho\left(g^{n}(z), g^{n}(x)\right) \rightarrow 0$ as $n \rightarrow \infty$; the local stable set $W_{\text {loc }}^{s}(z)$ of $z$ (of size $\beta$ ) is the set of points $x$ in $W^{s}(z)$ such that $\rho\left(F^{n}(z), F^{n}(x)\right) \leqq \beta$ for all integers $n \geqq 0$, where $\beta>0$. If $g=F$, then the (local) stable set is also called a (local) stable manifold. Notice that when $g=f$, and $f$ is expanding at such a point $z$, then the stable set of $z$ is the set of points that will be mapped into $z$ after a finite number of iterates (i.e., $W^{s}(z)=\left\{x: f^{n}(x)=z\right.$ for some integer $\left.n \geqq 0\right\}$ ). When $g=F$, the unstable manifold $W^{u}(z)$ of $z$ is the set of points $x$ for which $\rho\left(F^{-n}(z), F^{-n}(x)\right) \rightarrow 0$ as $n \rightarrow \infty$; and the local unstable manifold $W_{\mathrm{loc}}^{u}(z)$ of $z$ is the set of points $x$ in $W^{u}(z)$ such that $\rho\left(F^{-n}(z), F^{-n}(x)\right) \leqq \beta$ for all $n \geqq 0$, where $\beta>0$. If the stable or unstable 
manifold of $z \in \Omega(F)$ is a curve, we write $W_{\text {loc }}^{\sigma+}(z)$ and $W_{\text {loc }}^{\sigma-}(z)$ for the two components of $W_{\text {loc }}^{\sigma}(z) \backslash\{z\}$, where $\sigma$ is either $s$ or $u$. We will see below that the structure of the basin boundary is essentially controlled by finite sets of periodic points.

Recall that the intersection of the nonwandering set $\Omega(g)$ with the basin boundary BB can uniquely be decomposed into a finite collection of basic sets. From now on, let $\Gamma$ denote a basic set of $g$ that is contained in the basin boundary. We call $\Gamma$ a trivial basic set if $\Gamma$ consists of one periodic orbit, and we call $\Gamma$ a nontrivial basic set if $\Gamma$ includes more than one periodic orbit. If $\Gamma$ is nontrivial, then we call $\Gamma$ periodic if there exists a positive integer $m$ such that $g^{m}$ has no dense orbit on $\Gamma$, and otherwise we call $\Gamma$ nonperiodic.

We will often write dim instead of the notations $d_{b}, d_{H}$ and $d_{u}$, and we often refer to "dimension" rather than to "box-counting dimension", "uncertainty dimension" or "Hausdorff dimension". In such cases the corresponding statement is meant to apply for all three $d_{b}, d_{H}$ and $d_{u}$. Recall that for each point $x$ we write $B(x ; \varepsilon)$ for a ball with radius $\varepsilon$ centered at $x$, and that the pointwise dimensions $\operatorname{dim} \operatorname{BB}(x)$ of the basin boundary at $x$ is defined by

$$
\operatorname{dim} \mathrm{BB}(x)=\lim _{\varepsilon \rightarrow 0} \operatorname{dim}(B(x ; \varepsilon) \cap \mathrm{BB}) .
$$

Let $v$ be the number of basic sets in the basin boundary BB, and call these basic sets $\Gamma_{k}, 1 \leqq k \leqq v$.

Proposition 4-1. (i) Every point on the basin boundary BB is contained in the stable set of some nonwandering point in $\mathrm{BB}$. In other words, the basin boundary is the union of the stable sets $W^{s}\left(\Gamma_{k}\right), 1 \leqq k \leqq v$.

(ii) For every nonwandering point $z$ on the basin boundary, for each point $x$ in the stable set of $z$, the pointwise dimension of the basin boundary at $x$ and $z$ are equal, that is, $\operatorname{dim} \mathrm{BB}(x)=\operatorname{dim} \mathrm{BB}(z)$.

(iii) For each basic set $\Gamma_{k}(1 \leqq k \leqq v)$, all the points in $\Gamma_{k}$ have the same pointwise dimension, that is, $\operatorname{dim} \mathrm{BB}\left(z_{1}\right)=\operatorname{dim} \mathrm{BB}\left(z_{2}\right)$ for all $z_{1}, z_{2} \in \Gamma_{k}$.

(iv) The dimension of the basin boundary in a sufficiently small neighborhood of $\Gamma_{k}$ and the pointwise dimension at points of $\Gamma_{k}$ are equal.

Proof. For a proof of (i), (ii), and (iii), see [GNOY]. The proof of (iv) is left to the reader.

We write $d_{k}$ for the value of the pointwise dimension of the basin boundary at points in $\Gamma_{k}$. Note that the value $d_{k}$ in principle depends on which dimension we are using. We will prove that they are all equal, that is, $d_{b} \mathrm{BB}(z)=d_{u} \mathrm{BB}(z)=$ $d_{H} \mathrm{BB}(z)=d_{k}$, where $z \in \Gamma_{k}, 1 \leqq k \leqq v$.

We say that $x$ in the basin boundary is accessible from an open set $V$ if there is a curve $\gamma$ ending at $x$ such that all of $\gamma$ except for $x$ lies in $V$. We call $\gamma$ an access curve. For basin boundaries that are fractal, many points are not accessible from any of the basins. Note that for a Cantor set $C$ in $\mathbb{R}^{1}$, only countably many points of $C$ are accessible from the complement $\mathbb{R}^{1} \backslash C$.

If $x \in \Omega(F)$ is accessible from either $M \backslash W^{s}(\Omega(F))$ or $M \backslash W^{u}(\Omega(F))$, then a result due to Newhouse and Palis [NP] yields that $x$ is periodic. If we choose $V$ to be basin $\{A\}$ (for some attractor $A$ ), and if $x$ is on the basin boundary and is a nonwandering point, that is, $x \in \Omega(g) \cap \mathrm{BB}$, and if $x$ is accessible from basin $\{A\}$ and if $g=F$, then it is always possible to choose this curve $\gamma$ to be a piece of the 
unstable manifold $W^{u}(x)$, that is, either $W_{\text {loc }}^{u+}(x)$ or $W_{\text {loc }}^{u-}(x)$ is an acceptable choice for the access curve $\gamma$. Notice if $x$ is accessible from basin $\{A\}$ and the access curve $\gamma$ is $W_{\text {loc }}^{u+}(x)$, then $\gamma$ intersects $\Omega(F)$ only at $x$, so $x$ is not a limit point of $W_{\text {loc }}^{u+}(x) \cap \Omega(F)$. Applying a result due to Newhouse and Palis [NP], we obtain the following. The periodic points on the basin boundary that are accessible from $M \backslash W^{s}(\Omega(F))$ is a finite set, which we denote by $P^{u}$, and each point in BB that is accessible from $M \backslash W^{s}(\Omega(F))$ is in $W^{s}(p)$ fo some $p$ in $P^{u}$. Similarly, the periodic points on the basin boundary that are accessible from $M \backslash W^{u}(\Omega(F))$ is a finite set, say $P^{s}$, and each point in BB that is accessible from $M \backslash W^{u}(\Omega(F))$ is in $W^{u}(p)$ for some $p$ in $P^{s}$.

We say $R$ is a block for $\Gamma$, where $\Gamma$ is a nontrivial basic set, if (1) $R$ is a diffeomorphic image of the square $B=[-1,1] \times[-1,1]$, and (2) the four pieces of $\partial R$ are connected subsets of the stable manifolds of periodic points in $P^{u} \cap \Gamma$ and of the unstable manifolds of periodic points in $P^{s} \cap \Gamma$. Write $\partial_{s} R$ for the two segments of $\partial R$ that are segments of stable manifolds and $\partial_{u} R$ for the other two segments that are segments of unstable manifolds.

Palis and Takens [PT] have shown that for each nontrivial basic set $\Gamma$ there exists a finite collection of blocks for $\Gamma$ such that the blocks intersected with $\Gamma$ form a Markov partition. See Bowen [B] for the notion of Markov partition.

We say, a block partition for a nontrivial basic set $\Gamma$ is a finite collection $\left\{R_{i}\right\}_{i=1}^{N}$ of blocks for $\Gamma$ with $\Gamma \subset \bigcup_{i=1}^{N} R_{i}$.

Proposition 4-2. Assume that $\Gamma$ is a nontrivial nonperiodic (saddle-hyperbolic) basic set, and let $z \in \Gamma$ be fixed. There exist a block partition $\left\{R_{i}\right\}_{i=1}^{N}$ for $\Gamma$ and a connected subset $I^{u}$ of $W^{u}(z)$ such that for each $i$

(1) $I^{u} \cap R_{i}$ consists of exactly one component, and

(2) both points of $\partial\left(I^{u} \cap R_{i}\right)$ are in $\bigcup_{j=1}^{N} \partial_{s} R_{j}, 1 \leqq i \leqq N$.

Proof. For a proof, see Palis and Takens [PT].

Let $\Gamma$ be a nontrivial nonperiodic basic set. There exist a $C^{1+\alpha}$ stable foliation $\mathscr{F}^{s}$ on a neighborhood $V_{\Gamma}$ of $\Gamma$ and a $C^{1+\alpha}$ unstable foliation $\mathscr{F}^{u}$ on a neighborhood $V_{\Gamma}$ of $\Gamma$, for some $\alpha>0$. From now on, let the point $z \in \Gamma$, the blocks $R_{i}$, $1 \leqq i \leqq N$, and the segment $I^{u} \subset W^{u}(z)$ be as in Proposition 4-2. We can assume without loss of generality that the blocks are chosen small enough that all lie in $V_{\Gamma}$, see $[\mathrm{PT}]$.

Let $\tau: \mathbb{R} \rightarrow W^{u}(z)$ be a $C^{3}$ parametrization, and define a projection $\pi: \bigcup_{i=1}^{N} R_{i} \rightarrow \bigcup_{i=1}^{N} R_{i} \cap I^{u}$ by collapsing each $R_{i}$ onto $R_{i} \cap I^{u}$, projection along the local stable manifolds of the stable foliation $\mathscr{F}^{s}$. The following result says that for some iterate $K$, the map $F$ can be viewed as expansive along unstable segments.

Proposition 4-3. There exist a block partition $\left\{R_{i}\right\}_{i=1}^{N}$, an integer $K>0$, and a $C^{1+\alpha}$ map $\varphi: \bigcup_{i=1}^{N} \tau^{-1}\left(\pi R_{i}\right) \rightarrow \mathbb{R}$ defined by $\varphi(x)=\tau^{-1} \circ \pi \circ F^{K} \circ \tau(x)$ such that $\left|\varphi^{\prime}(x)\right|$ $>1$, for some $\alpha>0$.

Proof. For a proof, see Palis and Takens [PT].

4-2. Proofs of the One-Dimensional Results. In this subsection, we prove the theorem for one dimensional maps; along the way we present in Sect. 4-2A several auxiliary results that we use in the proof. The proofs of these results are either rather technical or similar to existing proofs in the literature; in the latter case we 
refer the reader to the literature. The reader is advised to skip the proofs of the intermediate results (which are given in Sect. 4-2B) with the first reading, and continue with the proof of the theorem that is presented in Sect. 4-2C.

4-2A. Statement of the One-Dimensional Auxiliary Results. The first result says that there exists a set $J$, which is a finite union of intervals, such that $f$ is expanding on $J$ and $J$ includes all the nonwandering points that are in the basin boundary.

Proposition 4-4. There exist $a$ set $J$ and an integer $L \geqq 1$ such that the following properties hold:

(1) $J$ is a finite union of open intervals;

(2) $\mathrm{BB} \cap \Omega(f) \subset J$;

(3) each component of $J$ intersects $\mathrm{BB} \cap \Omega(f)$;

(4) $f(J) \supset J$;

(5) $J$ contains no critical points and no images of critical points;

(6) $\inf _{J}\left|\left(f^{L}\right)^{\prime}(x)\right|>1$;

(7) there exists a $C>0$ such that if $x, y \in J$ and both $f^{i}(x)$ and $f^{i}(y)$ are in the same component of $J$ for each $i(0 \leqq i \leqq n)$, then $C^{-1} \leqq\left|\left(f^{n}\right)^{\prime}(x)\right| /\left|\left(f^{n}\right)^{\prime}(y)\right| \leqq C$.

Remark. If a set $J^{\#}$ satisfies (1),(2), (3) and (4) of Proposition 4-4 (when $J^{\#}$ replaces $J$ ), and $J^{\#} \subset J$, then (5), (6) and (7) hold trivially.

Let $\Gamma_{1}, \ldots, \Gamma_{s}$ be the basic sets of $f$ in the basin boundary, and let $J$ be as in Proposition 4-4. For each $k(1 \leqq k \leqq s)$, we write $J\left(\Gamma_{k}\right)$ for the union of components of $J$ that intersect $\Gamma_{k}$.

Lemma 4-5. The set $J$ in Proposition 4-4 can be chosen such that for all $i \neq k$, the closures of $J\left(\Gamma_{i}\right)$ and $J\left(\Gamma_{k}\right)$ are disjoint.

Let from now on, the sets $J\left(\Gamma_{k}\right), 1 \leqq k \leqq s$, are disjoint as in Lemma 4-5, and we call any such a set $J\left(\Gamma_{k}\right)$ an isolating neighborhood of $\Gamma_{k}$. For $1 \leqq k \leqq s$, the stable set $W^{s}\left(\Gamma_{k}\right)$ of $\Gamma_{k}$ is the union of the stable sets of points in $\Gamma_{k}$, that is, $W^{s}\left(\Gamma_{k}\right)=\bigcup_{z \in \Gamma_{k}} W^{s}(z)$. We say $\Gamma_{i} \leqq \Gamma_{j}$ if the union of the forward images $\bigcup_{n \geqq 0} f^{n}\left(J\left(\Gamma_{j}\right)\right)$ intersects $J\left(\Gamma_{i}\right)$. Equivalently, we say $\Gamma_{i} \leqq \Gamma_{j}$ if for each open neighborhood $V$ of $\Gamma_{j}$ the stable set $W^{s}\left(\Gamma_{i}\right)$ intersects $V$ (which implies that $W^{s}\left(\Gamma_{i}\right)$ intersects the unstable set $\left.W^{u}\left(\Gamma_{j}\right)\right)$.

Lemma 4-6. (Transitivity) The basic sets in the basin boundary can be indexed such that for all $i, j, k(1 \leqq i, j, k \leqq s)$ if both $\Gamma_{i} \leqq \Gamma_{j}$ and $\Gamma_{j} \leqq \Gamma_{k}$ then $\Gamma_{i} \leqq \Gamma_{k}$.

The next result says that the dimension of the basin boundary in the isolating neighborhood $J\left(\Gamma_{k}\right)$ of any basic set $\Gamma_{k}$ in the basin boundary equals the maximum value of the dimension of the basin boundary in the isolating neighborhoods $J\left(\Gamma_{j}\right)$ of $\Gamma_{j}$ for all $j(k \leqq j \leqq s)$ such that the union of the forward images $J\left(\Gamma_{j}\right)$ while iterating $f$, does intersect $J\left(\Gamma_{k}\right)$. Here, "dimension" refers to any of the three concepts of dimension in this paper.

Proposition 4-7. For each integer $k(1 \leqq k \leqq s)$, we have

$$
\operatorname{dim}\left(J\left(\Gamma_{k}\right) \cap \mathrm{BB}\right)=\max _{\Gamma_{k} \leqq \Gamma_{j}} \operatorname{dim}\left(J\left(\Gamma_{j}\right) \cap \mathrm{BB}\right) .
$$


The next two results say that the Hausdorff dimension, the box-counting dimension, and the uncertainty dimension of the basin boundary in the isolating neighborhood $J\left(\Gamma_{k}\right)$ of any basic set $\Gamma_{k}$ in the basin boundary are all three equal. To obtain our desired results for one dimensional maps, we must show the equality when arbitrary open intervals replace $J\left(\Gamma_{k}\right)$ below.

Proposition 4-8. For each integer $k(1 \leqq k \leqq s)$, we have

$$
d_{H}\left(J\left(\Gamma_{k}\right) \cap \mathrm{BB}\right)=d_{b}\left(J\left(\Gamma_{k}\right) \cap \mathrm{BB}\right) .
$$

Proposition 4-9. For each integer $k(1 \leqq k \leqq s)$, we have

$$
d_{\mathrm{H}}\left(J\left(\Gamma_{k}\right) \cap \mathrm{BB}\right)=d_{u}\left(J\left(\Gamma_{k}\right) \cap \mathrm{BB}\right) .
$$

4-2B. Proofs of the One-Dimensional Auxiliary Results. Let $U$ be the union of the basins, that is, $U=\bigcup_{k=1}^{q}$ basin $\left\{A_{k}\right\}$, so its components are open intervals. Let $I_{1}, \ldots, I_{w}$ be those (finitely many) components that intersect the attractors $\bigcup_{k=1}^{q} A_{k}$. For example, if $f$ is an Axiom $A$ map, then $I_{1}, \ldots, I_{w}$ are open intervals such that (1) $\bigcup_{k=1}^{w} I_{k}$ contains $\Omega_{a}(f)$ in its interior and (2) each $I_{k}$ includes a stable periodic point in its interior, $1 \leqq k \leqq w$.

From now on, we write $D_{0}$ for $\bigcup_{k=1}^{w} I_{k}$, and $B_{1}$ for the complement of $D_{0}$ in $M$, that is, $B_{1}=M \backslash D_{0}$. Notice that the definitions above imply $f\left(D_{0}\right) \subset D_{0}$ and $f\left(B_{1}\right) \supset B_{1}$. For every integer $n \geqq 0$ and each subset $S$ of $M$, we define the set $f^{-n}(S)=\left\{x \in M: f^{n}(x) \in S\right\}$.

For each integer $k \geqq 1$, we define

$$
B_{k+1}=M \backslash f^{-k}\left(D_{0}\right), \quad \text { and } \quad D_{k}=B_{k} \backslash B_{k+1} .
$$

Since $f\left(D_{0}\right) \subset D_{0}$, the sets $B_{k}$ are (decreasing) nested sets. The sets $B_{k+1}$ and $D_{k}$ arise naturally as follows. First, we define the sets of points that are mapped into $D_{0}$ and stay outside $D_{0}$ respectively, after iterating the map $f$ exactly $k$ times. The set $B_{k+1}$ is the set of points that will stay outside $D_{0}$ when $f$ is iterated $k$ times, and $D_{k}$ is the set of points that stay outside $D_{0}$ when $f$ is iterated less than $k$ times and are mapped into $D_{0}$ when $f$ is iterated $k$ times. This implies $D_{k}=\left\{x \in B_{k}: f^{k}(x) \in D_{0}\right\}$ $=B_{k} \backslash B_{k+1}$, and $B_{k+1}=\left\{x \in B_{k}: f^{k}(x) \in B_{1}\right\}=M \backslash \bigcup_{n}^{k}={ }_{0} f^{-n}\left(D_{0}\right)$.

Furthermore, we define

$$
B_{\infty}=\bigcap_{k=1}^{\infty} B_{k} .
$$

If an open interval $I$ includes points of the basin boundary but no nonwandering points, then after finitely many iterates, say $n$, the interval $f^{n}(I)$ will intersect the nonwandering set. For a proof of such a fact, see for example [Nu1, Nu2]. To prove that the dimensions are equal on an interval $S$, it is sufficient to prove it for $f(S)$, provided no critical points of $f$ are in $f(S)$. Continue iterating $f$, (assuming no critical points of $f$ are encountered) until $f^{k}(S)$ contains a point of $\Omega(f)$. We will show that it is sufficient to consider those components of the $B_{k}$ 's that intersect $\Omega(f)$. Therefore, for every $k \in \mathbb{N}$, we define the set

$$
B_{k}^{\circ}=\left\{x \in U: U \text { is a component of } B_{k} \text { and } U \cap \Omega(f) \neq \emptyset\right\} .
$$


By Lemma 3-5 in [Nu2] we know that there exist positive integers $L$ and $Q$ such that $\inf \left|\left(f^{L}\right)^{\prime}(x)\right|>1$, where the infimum is taken over all $x \in B_{Q}^{\circ}$. We fix $L$ and $Q$ for future reference.

Let $D_{0}^{*} \subset D_{0}$ be a closed set in $M$ with the following properties: (1) $D_{0}^{*}$ intersects each component $D$ of $D_{0} ;(2)$ the interior $\operatorname{Int}\left(D_{0}^{*}\right)$ of $D_{0}^{*}$ includes the attractors $\bigcup_{k=1}^{q} A_{k} ;(3) f\left(D_{0}^{*}\right) \subset \operatorname{Int}\left(D_{0}^{*}\right) ;(4)$ if a critical point $c$ of $f$ satisfies $f^{n}(c) \in$ $D_{0}$, for some nonnegative integer $n$, then $f^{n}(c) \in \operatorname{Int}\left(D_{0}^{*}\right) ;(5)$ the number of the components of $D_{0}^{*}$ is equal to the number of components of $D_{0}$.

We define for each integer $k \geqq 0$, the sets $D_{k}^{*}=f^{-k}\left(D_{0}^{*}\right)$, and $J_{k}$ is the union of those components of $M \backslash D_{k}^{*}$ which intersect both $\Omega(f)$ and BB.

Lemma 4-10. The number $R_{0}=\min \left\{n \in \mathbb{N} \cup\{0\}: J_{n}\right.$ includes no critical point of $f\}$ is well defined, and $R_{0} \leqq Q$. For every integer $n \geqq R_{0}$, the set $J_{n}$ has the following properties:

(i) $J_{n}$ is open in $I$;

(ii) the restriction of $f$ to $J_{n}$ is a homeomorphism on each interval in $J_{n}$;

(iii) $J_{n}$ has finitely many components.

Proof. The proof is similar to the proof of Lemma 3-7 in [Nu2].

We will assume that $D_{0}^{*}$ is chosen such that inf $\left|\left(f^{L}\right)^{\prime}(x)\right|>1$, where the infimum is taken over all $x$ in the closure of $J_{Q}$ (note that this assumption is no restriction). We obtain that $\bar{J}_{k}$ (the closure of $J_{k}$ ) are nested (decreasing) compact intervals, and $f\left(J_{k}\right) \supset J_{k}$.

Lemma 4-11. There exists $C>0$ such that for each $n \in \mathbb{N} \cup\{0\}$, for each component $U$ in $J_{Q+n}$ one has

$$
C^{-1} \leqq\left|\left(f^{n}\right)^{\prime}(x)\right| /\left|\left(f^{n}\right)^{\prime}(y)\right| \leqq C, \text { for all } x, y \text { in } U
$$

Proof. We write $G=f^{L}$. There exists $K>0$ such that the distortion inequality $K^{-1} \leqq\left|\left(G^{T}\right)^{\prime}(x)\right| /\left|\left(G^{T}\right)^{\prime}(y)\right| \leqq K$ holds, for all $T \geqq 0$, and all $x, y$ in the same component of $J_{T L+Q}$, since $\left|\left(f^{L}\right)^{\prime}(x)\right|>1$ for each $x \in J_{Q}$ (see e.g. [Ma]). Now we define $E=\max \left\{\max \left\{\left|f^{\prime}(x)\right|: x \in \bar{J}_{Q}\right\}, \min \left\{\left|f^{\prime}(x)\right|^{-1}: x \in \bar{J}_{Q}\right\}\right\}$, where $\bar{J}_{Q}$ is the closure of $J_{Q}$.

Selecting $C=K \cdot E^{L}$ we obtain for every nonnegative integer $n, C^{-1} \leqq$ $\left|\left(f^{n}\right)^{\prime}(x)\right| /\left|\left(f^{n}\right)^{\prime}(y)\right| \leqq C$, for all $x, y$ in $U$, where $U$ is an arbitrary component in $J_{Q+n}$.

Proof of Proposition 4-4. Let the integers $Q$ and $L$ be as above. We select the set $J$ to be $J_{Q}$.

(1) Lemma 4-10 yields $J_{Q}$ consists of finitely many open intervals, hence $J$ is a finite union of open intervals.

(2) By definition we have that $J_{Q}$ includes $\mathrm{BB} \cap \Omega(f)$, and so $J$ includes $\mathrm{BB} \cap \Omega(f)$.

(3) By definition each component of $J_{Q}$ intersects $\mathrm{BB} \cap \Omega(f)$. Therefore, each component of $J$ intersects $\mathrm{BB} \cap \Omega(f)$.

(4) Since $f\left(J_{Q}\right) \supset J_{Q}$ we have $f(J) \supset J$.

(5) The property inf $\left|\left(f^{L}\right)^{\prime}(x)\right|>1$ (where the infimum is taken over all $x$ in the closure of $J_{Q}$ ) implies $J$ contains no critical points and no images of critical-points. 
(6) The property $\inf \left|\left(f^{L}\right)^{\prime}(x)\right|>1$ (where the infimum is taken over all $x$ in the closure of $\left.J_{Q}\right)$ yields inf $\operatorname{in}_{J}\left|\left(f^{L}\right)^{\prime}(x)\right|>1$.

(7) Let $C>0$ be a constant as in Lemma 4-11. Let integer $n \geqq 0$ be fixed. Let $x, y \in J$ and assume that both $f^{i}(x)$ and $f^{i}(y)$ are in the same component of $J$ for each $i, 0 \leqq i \leqq n$. This assumption implies that $x$ and $y$ are in the same component of $J_{Q+n}$. Now we apply Lemma 4-11 and obtain $C^{-1} \leqq\left|\left(f^{n}\right)^{\prime}(x)\right| /\left|\left(f^{n}\right)^{\prime}(y)\right|$ $\leqq C$.

Proof of Lemma 4-5. Recall that $f$ is hyperbolic on the basin boundary and that $\Gamma_{1}, \ldots, \Gamma_{s}$ are the basic sets in $\mathrm{BB} \cap \Omega(f)$. Let $V_{k}(1 \leqq k \leqq s)$ be an open neighborhood of $\Gamma_{k}$ such that for all $i \neq k$ the neighborhoods $V_{i}$ and $V_{k}$ are disjoint. Such neighborhoods exist, because the basic sets $\Gamma_{k}$ are disjoint, compact sets. In a similar way as Lemma 5-6 in [Nu2] has been proved, one shows that the total length of $J_{k}$ goes to zero as $k \rightarrow \infty$. Select integer $n \geqq R_{0}$ in Lemma 4-10 such that the closure of $J_{n}$ is contained in the open neighborhood $\bigcup_{k} V_{k}$ of BB $\cap \Omega(f)$. The conclusion now is that for all $i \neq k$, the closures of $J\left(\Gamma_{i}\right)$ and $J\left(\Gamma_{k}\right)$ are disjoint.

Let $\tilde{N}$ denote the number of disjoint components $J_{Q}^{i}$ of $J_{Q}$, that is, $J_{Q}=\bigcup_{i=1}^{\tilde{N}} J_{Q}^{i}$. We define the $\tilde{N} \times \tilde{N}$ matrix $A$ by

$$
A(i, j)=\left\{\begin{array}{ll}
1 & \text { if } f\left(J_{Q}^{i}\right) \supset J_{Q}^{j} \\
0 & \text { otherwise }
\end{array} \quad 1 \leqq i, j \leqq \tilde{N} .\right.
$$

We will assume that the $J_{Q}^{i}$ 's are numbered in such a way that the matrix $A$ is written in the form, see [BP]

$$
A=\left[\begin{array}{ccccc}
A_{11} & 0 & \cdots & \cdots & 0 \\
A_{21} & A_{22} & \ddots & \vdots \\
\vdots & & \ddots & \ddots & \vdots \\
\vdots & & \ddots & \ddots \\
\vdots & & \ddots & 0 \\
A_{s 1} & \cdots & \cdots & \ddots & A_{s s}
\end{array}\right],
$$

where each block $A_{k k}(1 \leqq k \leqq s)$ is an irreducible $N_{k} \times N_{k}$ matrix (that is, for each pair $(i, j)$ there exists an integer $n \geqq 1$ such that the $(i, j)^{\text {th }}$ entry (where $1 \leqq i, j \leqq N_{k}$ ) of the matrix $\left(A_{k k}\right)^{n}$ is positive), and $\sum_{k=1}^{s} N_{k}=\tilde{N}$ for some $s, 1 \leqq s \leqq \tilde{N}$. In fact, with the techniques in $[\mathrm{Nu} 1, \mathrm{Nu} 2]$ one can show that $1 \leqq s \leqq q+1$.

For each $k, 1 \leqq k \leqq s$, we write

$$
\mathscr{B}_{0}^{k}=\left\{\bigcup_{i} J_{Q}^{i}: \sum_{j=1}^{k-1} N_{j}<i \leqq \sum_{j=1}^{k} N_{j}\right\}
$$

(we define $\sum_{j=1}^{0} N_{j}=0$ ), and for every integer $n \geqq 0$ we define

$$
\mathscr{B}_{n}^{k}=\left\{x \in \mathscr{B}_{0}^{k}: f^{n}(x) \in \mathscr{B}_{0}^{k}\right\}
$$

or equivalently,

$$
\mathscr{B}_{n}^{k}=f^{-n}\left(\mathscr{B}_{0}^{k}\right) \cap \mathscr{B}_{0}^{k}
$$

and finally,

$$
\mathscr{B}_{\infty}^{k}=\bigcap_{n=0}^{\infty} \mathscr{B}_{n}^{k}
$$


For every (irreducible) matrix $A_{k k}$ and for each $n$, the set $\mathscr{B}_{n}^{k}$ has finitely many components, say $N\left(\mathscr{B}_{n}^{k}\right)$, and we write $\mathscr{B}_{n}^{k}$ as the disjoint union of the components $\mathscr{B}_{n ; i}^{k}, 1 \leqq i \leqq N\left(\mathscr{B}_{n}^{k}\right)$.

Proof of Lemma 4-6. Lemma 4-5 and the definition of the sets $\mathscr{B}_{n}^{k}$ imply that each set $\mathscr{B}_{n}^{k}$ includes one basic set of BB $\cap \Omega(f)$. Hence, after possibly relabeling the basic sets, we have $\Gamma_{k} \subset \mathscr{B}_{n}^{k}$ for all positive integers $n$. Let integers $i, j, k$ $(1 \leqq i, j, k \leqq s)$ be given, and assume that both $\Gamma_{i} \leqq \Gamma_{j}$ and $\Gamma_{j} \leqq \Gamma_{k}$.

Let $G(A)$ be the directed graph associated with the matrix $A$, that is, the graph $G(A)$ consists of the vertices labeled $J_{Q}^{i}, 1 \leqq i \leqq \tilde{N}$ and a number of directed edges, namely there is a directed edge from vertex $J_{Q}^{i}$ to vertex $J_{Q}^{j}$ if and only if $A(i, j)=1$. Let $I_{i}, I_{j}$, and $I_{k}$ be any interval in $\mathscr{B}_{0}^{i}, \mathscr{B}_{0}^{j}$, and $\mathscr{B}_{0}^{k}$ respectively. The assumption that both $\Gamma_{i} \leqq \Gamma_{j}$ and $\Gamma_{j} \leqq \Gamma_{k}$ and the fact that there is a path from $I_{r}$ to any other interval in $\mathscr{B}_{0}^{r}$ (where $r=i, j, k$ ) imply that there is both a path from $\Gamma_{j}$ to $\Gamma_{i}$, and a path from $\Gamma_{k}$ to $\Gamma_{j}$. Hence $\Gamma_{i} \leqq \Gamma_{k}$.

Proof of Proposition 4-7. Proposition 4-1, Proposition 4-4, and Lemma 4-6 yield the desired result.

Proposition 4-12. $d_{H}\left(\mathscr{B}_{\infty}^{k}\right)=d_{b}\left(\mathscr{B}_{\infty}^{k}\right)$, for all $1 \leqq k \leqq s$.

Proof. From the fact that $f^{L}$ is expanding, and by using the result due to Takens [T] we obtain immediately that $d_{H}\left(\mathscr{B}_{\infty}^{k} \cap \Omega\left(f^{L}\right)\right)=d_{b}\left(\mathscr{B}_{\infty}^{k} \cap \Omega\left(f^{L}\right)\right)$. This result together with the fact $\mathscr{B}_{\infty}^{k} \cap \Omega\left(f^{L}\right)=\mathscr{B}_{\infty}^{k} \cap \Omega(f)$ and Proposition 4-1 yields the result.

Corollary 4-13. Fix any $k, 1 \leqq k \leqq s$. If $d>d_{H}\left(\mathscr{B}_{0}^{k}\right)$ then $\lim _{n \rightarrow \infty} \sum_{i=1}^{N\left(\mathscr{B}_{n}^{k}\right)}\left|\mathscr{B}_{n ; i}^{k}\right|^{d}=0$, and if $d<d_{H}\left(\mathscr{B}_{\infty}^{k}\right)$ then $\sum_{i=1}^{N\left(\mathscr{\mathscr { B }}_{n}^{k}\right)}\left|\mathscr{B}_{n ; i}^{k}\right|^{d} \rightarrow \infty$ when $n \rightarrow \infty$.

Proof. The definition of Hausdorff dimension together with Proposition 4-12 yields the result.

Proof of Proposition 4-8. Apply Proposition 4-1, Proposition 4-7 and Proposition 4-12 and obtain the desired result.

For each $k(1 \leqq k \leqq s)$ and for each integer $n \geqq 0$, we write $\varepsilon_{n}^{k}$ for the minimum of the minimum of the distance between two different components of $\mathscr{B}_{n}^{k}$ and the distance between the boundary of $\mathscr{B}_{n}^{k}$ and $\mathscr{B}_{\infty}^{k}$, that is,

$$
\varepsilon_{n}^{k}=\min \left\{\min _{i \neq j} \operatorname{dist}\left(\mathscr{B}_{n ; i}^{k}, \mathscr{B}_{n ; j}^{k}\right), \operatorname{dist}\left(\mathscr{B}_{\infty}^{k}, \operatorname{Bndy}\left(\mathscr{B}_{n}^{k}\right)\right)\right\} .
$$

Proposition 4-14. Let integer $k, 1 \leqq k \leqq s$, be given. Then $d_{H}\left(\mathscr{B}_{\infty}^{k} \cap \mathrm{BB}\right)$ $=d_{u}\left(\mathscr{B}_{\infty}^{k} \cap \mathrm{BB}\right)$.

Proof. Let $1 \leqq k \leqq s$ be given. It is left to the reader to prove $d_{H}\left(\mathscr{B}_{\infty}^{k} \cap \mathrm{BB}\right)=d_{u}\left(\mathscr{B}_{\infty}^{k} \cap \mathrm{BB}\right)$ when $\left(\mathscr{B}_{\infty}^{k} \cap \mathrm{BB}\right)$ is trivial, that is, $\left(\mathscr{B}_{\infty}^{k} \cap \mathrm{BB}\right)$ is a single periodic orbit. From now on, we assume $\left(\mathscr{B}_{\infty}^{k} \cap \mathrm{BB}\right)$ is nontrivial.

Let $\delta>d_{H}\left(\mathscr{B}_{\infty}^{k}\right)$, and let $L_{m}^{k}$ and $L_{M}^{k}$ denote the minimum and maximum length of the components of $\mathscr{B}_{0}^{k}$ respectively. By Corollary $4-13$, select $\mathscr{N}_{k} \in \mathbb{N}$ such that $\sum_{i=1}^{N\left(\mathscr{B}_{n}^{k}\right)}\left|\mathscr{B}_{n ; i}^{k}\right|^{\delta}<\left(C / L_{m}^{k}\right)^{-\delta}$ for every $n \geqq \mathscr{N}_{k}$, where $C$ is the constant in Proposition 4-4. Fix $n \geqq \mathscr{N}_{k}$; recall that $\varepsilon_{n}^{k}=\min \left\{\min _{i \neq j} \operatorname{dist}\left(\mathscr{B}_{n ; i}^{k}, \mathscr{B}_{n ; j}^{k}\right)\right.$, $\operatorname{dist}\left(\mathscr{B}_{\infty}^{k}\right.$, $\left.\left.\operatorname{Bndy}\left(\mathscr{B}_{n}^{k}\right)\right)\right\}$. For $0<\varepsilon<\varepsilon_{n}^{k}$, denote the probability that two points $x, y$ (chosen at 
random from $\mathscr{B}_{0}^{k}$ according to the uniform distribution and subject to the condition $|x-y|<\varepsilon)$ tend to different attractors by $p^{k}(\varepsilon)$. That is, let $I_{x}(\varepsilon)=[x-\varepsilon, x+\varepsilon]$, let $\mu_{1}$ denote the one dimensional Lebesgue measure, set

$$
p_{x}^{k}(\varepsilon)=\mu_{1}\left(\left\{y \in I_{x}(\varepsilon) \cap \mathscr{B}_{0}^{k}: L^{+}(x) \neq L^{+}(y)\right\}\right) / \mu_{1}\left(I_{x}(\varepsilon) \cap \mathscr{B}_{0}^{k}\right),
$$

and then

$$
p^{k}(\varepsilon)=\int_{\mathscr{B}_{n}^{k}} p_{x}^{k}(\varepsilon) d x
$$

In order to finish the proof it is sufficient to show that $\lim _{\varepsilon \rightarrow 0} \frac{\ln p^{k}(\varepsilon)}{\ln \varepsilon}=1-d_{H}\left(\mathscr{B}_{\infty}^{k}\right)$.

For every $\varepsilon, 0<\varepsilon<\varepsilon_{n}^{k}$, define the sets

$$
\begin{aligned}
W^{k}(\varepsilon) & =\left\{(x, y) \in \mathscr{B}_{0}^{k} \times \mathscr{B}_{0}^{k}:|x-y|<\varepsilon\right\}, \\
V^{k}(\varepsilon) & =\left\{(x, y) \in W^{k}(\varepsilon): L^{+}(x) \neq L^{+}(y)\right\},
\end{aligned}
$$

and denote by $\mu_{2}$ the two dimensional Lebesgue measure. Then we have $p^{k}(\varepsilon) \approx \mu_{2}\left(V^{k}(\varepsilon)\right) / \mu_{2}\left(W^{k}(\varepsilon)\right)$ for $\varepsilon$ sufficiently small. Since $\mu_{2}\left(W^{k}(\varepsilon)\right)=$ $\mu_{1}\left(\mathscr{B}_{0}^{k}\right) \cdot\left\{2 \varepsilon-\varepsilon^{2}\right\}$, we are done if we show that $\lim _{\varepsilon \rightarrow 0} \frac{\ln \mu_{2}\left(V^{k}(\varepsilon)\right)}{\ln \varepsilon}=2-d_{H}\left(\mathscr{B}_{\infty}^{k}\right)$.

For $0<\varepsilon<\varepsilon_{n}^{k}$, let $\mathfrak{C}_{\varepsilon}=\left\{C_{j}\right\}_{j \in \mathscr{J}}$ denote a cover of $\mathscr{B}_{\infty}^{k}$ by $\mathscr{N}^{k}(\varepsilon)$ sets with diameter $\varepsilon$; assume that $\mathscr{N}^{k}(\varepsilon)$ is minimal. For every $\varepsilon, 0<\varepsilon<\varepsilon_{n}^{k}$, the assumption on $\mathfrak{C}_{\varepsilon}$ that $\mathscr{N}^{k}(\varepsilon)$ is minimal yields each element of $\mathfrak{C}_{\varepsilon}$ is contained in $\mathscr{B}_{n}^{k}$. For a moment fix $\varepsilon, 0<\varepsilon<\varepsilon_{n}^{k}$, and some integer $i, 1 \leqq i \leqq N\left(\mathscr{B}_{n}^{k}\right)$; then, since $f^{n}\left(\mathscr{B}_{n ; i}^{k}\right)=\mathscr{B}_{0 ; m}^{k}$ for some $m$, the collection $\left\{f^{n}\left(C_{j} \cap \mathscr{B}_{n ; i}^{k}\right)\right\}_{j \in \mathscr{J}}$ is a cover of $\mathscr{B}_{0 ; m}^{k} \cap \mathscr{B}_{\infty}^{k}$, where $1 \leqq m \leqq N\left(\mathscr{B}_{0}^{k}\right)$. Applying Lemma 4-11 shows $C^{-1} \cdot \varepsilon^{*} \cdot$ $\left|\mathscr{B}_{n ; i}^{k}\right|^{-1}=C^{-1} \cdot\left|C_{j} \cap \mathscr{B}_{n ; i}^{k}\right| /\left|\mathscr{B}_{n ; i}^{k}\right| \leqq\left|f^{n}\left(C_{j} \cap \mathscr{B}_{n ; i}^{k}\right)\right| /\left|f^{n}\left(\mathscr{B}_{n ; i}^{k}\right)\right|=\left|f^{n}\left(C_{j} \cap \mathscr{B}_{n ; i}^{k}\right)\right| \mid$ $\left|\mathscr{B}_{0 ; m}^{k}\right| \leqq C \cdot\left|C_{j} \cap \mathscr{B}_{n ; i}^{k}\right| /\left|\mathscr{B}_{n ; i}^{k}\right|=C \cdot \varepsilon \cdot\left|\mathscr{B}_{n ; i}^{k}\right|^{-1} ; \quad$ therefore each element of $\left\{f^{n}\left(C_{j} \cap \mathscr{B}_{n ; i}^{k}\right)\right\}_{j \in \mathcal{J}}$ has diameter in the interval $\left[L_{m}^{k} \cdot C^{-1} \cdot \varepsilon \cdot\left|\mathscr{B}_{n ; i}^{k}\right|^{-1}\right.$, $\left.L_{M}^{k} \cdot C \cdot \varepsilon \cdot\left|\mathscr{B}_{n ; i}^{k}\right|^{-1}\right]$, where $L_{m}^{k}$ and $L_{M}^{k}$ are the minimum and maximum length of the components of $\mathscr{B}_{0}^{k}$ respectively.

Define the map $G: \mathscr{B}_{0}^{k} \times \mathscr{B}_{0}^{k} \rightarrow \mathbb{R}^{2}$ by $G(x, y)=(f(x), f(y))$. Obviously, if $(x, y) \in \mathscr{B}_{n ; i}^{k} \times \mathscr{B}_{n ; i}^{k}$ and $(w, z) \in \mathscr{B}_{n ; i}^{k} \times \mathscr{B}_{n ; i}^{k}$ for some $1 \leqq i \leqq N\left(\mathscr{B}_{n}^{k}\right)$, then $C^{-2} \leqq$ $\left|\operatorname{det} D G^{n}(x, y) / \operatorname{det} D G^{n}(w, z)\right| \leqq C^{2}$, where $C>0$ is the constant in Lemma 4-11.

For every integer $i, 1 \leqq i \leqq N\left(\mathscr{B}_{n}^{k}\right)$, and for all $\varepsilon, 0<\varepsilon<\varepsilon_{n}^{k}$ we have

$$
\begin{aligned}
& V^{k}\left(L_{m}^{k} \cdot C^{-1} \cdot \varepsilon \cdot\left|\mathscr{B}_{n ; i}^{k}\right|^{-1}\right) \subset G^{n}\left(V^{k}(\varepsilon) \cap\left(\mathscr{B}_{n ; i}^{k} \times \mathscr{B}_{n ; i}^{k}\right)\right), \\
& G^{n}\left(V^{k}(\varepsilon) \cap\left(\mathscr{B}_{n ; i}^{k} \times \mathscr{B}_{n ; i}^{k}\right)\right) \subset V^{k}\left(L_{M}^{k} \cdot C \cdot \varepsilon \cdot\left|\mathscr{B}_{n ; i}^{k}\right|^{-1}\right),
\end{aligned}
$$

where $L_{m}^{k}$, respectively $L_{M}^{k}$, is the minimum, respectively maximum, length of the components of $\mathscr{B}_{0}^{k}$.

Let $\varepsilon, 0<\varepsilon<\varepsilon_{n+N_{k}-1}^{k}$, be given. Note that $(x, y) \in V^{k}(\varepsilon)$ implies that one of $x, y$ lies in a component $\mathscr{B}_{n ; i}^{k}$, for some $1 \leqq i \leqq N\left(\mathscr{B}_{n}^{k}\right)$. For each integer $j, 1 \leqq j \leqq N\left(\mathscr{B}_{n}^{k}\right)$, we write $V_{n ; j}^{k}(\varepsilon)$ for the component of $V^{k}(\varepsilon)$ that has a nonempty intersection with $\mathscr{B}_{n ; j}^{k} \times \mathscr{B}_{n ; j}^{k}$, and we set $\mathscr{D}_{n ; j}^{k}(\varepsilon)=V_{n ; j}^{k}(\varepsilon) \backslash\left(\mathscr{B}_{n ; j}^{k} \times \mathscr{B}_{n ; j}^{k}\right)$. It now follows that $\cup \mathscr{D}_{n ; j}^{k}(\varepsilon)=\left(\bigcup_{j} \mathscr{D}_{n ; j}^{k}(\varepsilon)\right) \cap V^{k}(\varepsilon)$ is contained in the image under $G^{m}$ of a subset of $V^{k}(\varepsilon) \cap\left(\bigcup_{j}\left(\mathscr{B}_{n ; j}^{k} \times \mathscr{B}_{n ; j}^{k}\right)\right)$, where $m=N_{k}-1$. Hence, there exists 
a constant $\hat{A}>1$ such that $\mu_{2}\left(V^{k}(\varepsilon)\right) \leqq \hat{A} \cdot \mu_{2}\left(\bigcup_{j}\left\{V^{k}(\varepsilon) \cap\left(\mathscr{B}_{n ; j}^{k} \times \mathscr{B}_{n ; j}^{k}\right)\right\}\right)$. Now we apply $(1 \mathrm{a}, 1 \mathrm{~b})$, and summing over $i$ yields

$$
\begin{aligned}
& \mu_{2}\left(V^{k}(\varepsilon)\right) \geqq C^{-2} \cdot \sum_{i}\left|\mathscr{B}_{n ; i}^{k}\right|^{2} \cdot V^{k}\left(L_{m}^{k} C^{-1} \varepsilon \cdot\left|\mathscr{B}_{n ; i}^{k}\right|^{-1}\right), \\
& \mu_{2}\left(V^{k}(\varepsilon)\right) \leqq \hat{A} \cdot C^{2} \cdot \sum_{i}\left|\mathscr{B}_{n ; i}^{k}\right|^{2} \cdot V^{k}\left(L_{M}^{k} C^{-1} \varepsilon \cdot\left|\mathscr{B}_{n ; i}^{k}\right|^{-1}\right) .
\end{aligned}
$$

For $d \geqq 0$ define $\psi^{k}(\varepsilon)$ by $\mu_{2}\left(V^{k}(\varepsilon)\right)=\varepsilon^{d} \cdot \psi^{k}(\varepsilon)$. Assume first that $d<2-d_{H}\left(B_{\infty}^{k}\right)$. By Corollary $4-13$, select $n$ so large such that $\sum_{i}\left|\mathscr{B}_{n ; i}^{k}\right|^{2-d}<\left(\hat{A} \cdot C^{2}\right)^{-1}$. Substituting in (2b), for $\varepsilon$ sufficiently small, we obtain

$$
\begin{aligned}
\psi^{k}(\varepsilon) & \leqq \hat{A} \cdot C^{2} \sum_{i}\left|\mathscr{B}_{n ; i}^{k}\right|^{2-d} \cdot \psi^{k}\left(L_{M}^{k} C^{-1} \varepsilon \cdot\left|\mathscr{B}_{n ; i}^{k}\right|^{-1}\right) \\
& \leqq \sum_{i} \beta_{i} \cdot \psi^{k}\left(L_{M}^{k} \cdot C^{-1} \cdot \varepsilon \cdot\left|\mathscr{B}_{n ; i}^{k}\right|^{-1}\right), \quad \text { where } \sum_{i} \beta_{i}=1 .
\end{aligned}
$$

This expression gives a convex combination of values of $\psi^{k}$ at larger arguments (recall that $L_{M}^{k} \cdot C^{-1} \cdot\left|\mathscr{B}_{n ; i}^{k}\right|^{-1}>1$ ) as an upper bound for $\psi^{k}(\varepsilon)$, so $\psi^{k}(\varepsilon)$ is bounded. Hence, $\lim _{\varepsilon \rightarrow 0} \ln \mu_{2}\left(V^{k}(\varepsilon)\right) / \ln \varepsilon=\lim _{\varepsilon \rightarrow 0}\left\{d+\ln \psi^{k}(\varepsilon) / \ln \varepsilon\right\} \geqq d$.

Now assume that $d>2-d_{H}\left(B_{\infty}^{k}\right)$. By Corollary 4-13, select $n$ such that $\sum_{i}\left|\mathscr{B}_{n ; i}^{k}\right|^{2-d}>C^{2}$. Applying (2a), for $\varepsilon$ sufficiently small, we get

$$
\begin{aligned}
\psi^{k}(\varepsilon) & \geqq \hat{A} \cdot C^{-2} \sum_{i}\left|\mathscr{B}_{n ; i}^{k}\right|^{2-d} \cdot \psi^{k}\left(L_{m}^{k} C^{-1} \varepsilon \cdot\left|\mathscr{B}_{n ; i}^{k}\right|^{-1}\right) \\
& \geqq \sum_{i} \beta_{i} \cdot \psi^{k}\left(L_{m}^{k} \cdot C^{-1} \cdot \varepsilon \cdot\left|\mathscr{B}_{n ; i}^{k}\right|^{-1}\right), \quad \text { where } \sum_{i} \beta_{i}=1 .
\end{aligned}
$$

This expression is a convex combination of values of $\psi^{k}$ at larger arguments (recall $L_{m}^{k} \cdot C^{-1}\left|\mathscr{B}_{n ; i}^{k}\right|^{-1}>1$ ) as a lower bound for $\psi^{k}(\varepsilon)$, so $\psi^{k}(\varepsilon)$ is bounded away from zero. Hence,

$$
\lim _{\varepsilon \rightarrow 0} \sup \ln \mu_{2}\left(V^{k}(\varepsilon)\right) / \ln \varepsilon=d+\lim _{\varepsilon \rightarrow 0} \sup \ln \psi^{k}(\varepsilon) / \ln \varepsilon \leqq d .
$$

The conclusion is $\lim _{\varepsilon \rightarrow 0} \frac{\ln \mu_{2}\left(V^{k}(\varepsilon)\right)}{\ln \varepsilon}=2-d_{H}\left(\mathscr{B}_{\infty}^{k}\right)$.

Proof of Proposition 4-9. Apply Proposition 4-1, Proposition 4-7 and Proposition 4-14 yielding the desired result.

4-2C. Poof of the (One-Dimensional) Theorem. Assume that $f: M \rightarrow M$ has the following properties: (1) there exists finitely many, attractors, say $A_{1}, A_{2}, \ldots, A_{q}$ where $q \geqq 2$, (2) for each point $x \in M$, there exists an integer $k, 1 \leqq k \leqq q$, such that either $x \in$ basin $\left\{A_{k}\right\}$ or $x$ is on the boundary of basin $\left\{A_{k}\right\}$, and (3) $f$ is hyperbolic on the basin boundary BB. Let $S$ be an arbitrarily given region that intersects the basin boundary.

Let the integer $s \geqq 1$, and the sets $\mathscr{B}_{n}^{k}$ and $\mathscr{B}_{\infty}^{k}$, where $1 \leqq k \leqq s$ and $n \geqq 0$ are integers, be as in Sect. 4-2B.

For every $k, 1 \leqq k \leqq s$, we define $\mathscr{B}_{\infty}^{k}(S)=\mathscr{B}_{\infty}^{k} \quad$ if $\quad S$ intersects $\left(\bigcup_{n=0}^{\infty} f^{-n}\left(\mathscr{B}_{\infty}^{k}\right)\right) \cap \mathrm{BB}$, and $\mathscr{B}_{\infty}^{k}(S)=\emptyset$ otherwise. Recall that both the Hausdorff dimension and the box-counting dimension of an empty set are defined to be zero. From elementary properties of Hausdorff dimension and the box- 
counting dimension it follows that

$$
\begin{aligned}
& d_{b}\left(\bigcup_{k=1}^{s} \mathscr{B}_{\infty}^{k}(S)\right)=\max _{1 \leqq k \leqq s} d_{b}\left(\mathscr{B}_{\infty}^{k}(S)\right), \quad \text { and } \\
& d_{H}\left(\bigcup_{k=1}^{s} \mathscr{B}_{\infty}^{k}(S)\right)=\max _{1 \leqq k \leqq s} d_{H}\left(\mathscr{B}_{\infty}^{k}(S)\right) .
\end{aligned}
$$

This together with the combinatorial results in [Nu1, Nu2] and Propositions 4-1, 4-7 and 4-8 imply $d_{b}(S \cap \mathrm{BB})=d_{H}(S \cap \mathrm{BB})$.

For every integer $k, 1 \leqq k \leqq s$, for each integer $n \geqq 0$ let the number $\varepsilon_{n}^{k}>0$ and the integer $\mathscr{N}_{k} \geqq 1$ be defined as in the proof of Proposition 4-14. Fix any

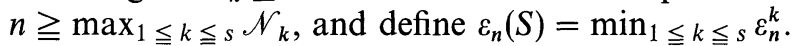

For $0<\varepsilon<\varepsilon_{n}(S)$, denote the probability that two points $x, y$ (chosen at random from $S$ according to the uniform distribution and subject to the condition $|x-y|<\varepsilon)$ tend to different attractors by $p(\varepsilon, S)$. That is, let $I_{x}(\varepsilon)=[x-\varepsilon, x+\varepsilon]$, let $\mu_{1}$ denote the one dimensional Lebesgue measure, set

$$
p_{x}(\varepsilon, S)=\mu_{1}\left(\left\{y \in I_{x}(\varepsilon) \cap S: L^{+}(x) \neq L^{+}(y)\right\}\right) / \mu_{1}\left(I_{x}(\varepsilon) \cap S\right),
$$

and then

$$
p(\varepsilon, S)=\int_{S} p_{x}(\varepsilon, S) d x
$$

For every integer $k, 1 \leqq k \leqq s$, for each $\varepsilon>0$, for each $x \in S$, define $p_{x}^{k}(\varepsilon, S)=p_{x}^{k}(\varepsilon)$ and $p^{k}(\varepsilon, S)=p^{k}(\varepsilon)$ if the intersection $S \cap\left(\bigcup_{n=0}^{\infty} f^{-n}\left(\mathscr{B}_{\infty}^{k}\right)\right) \cap \mathrm{BB}$ is nonempty, and $p_{x}^{k}(\varepsilon, S)=p^{k}(\varepsilon, S)=0$ otherwise. Finally, we define $p_{x}^{k}(\varepsilon, S) \cdot\left(\mu_{1}\left(I_{x}(\varepsilon) \cap \mathscr{B}_{0}^{k}(S)\right) / \mu_{1}\left(I_{x}(\varepsilon) \cap S\right)\right)=0 \quad$ if $S \cap\left(\bigcup_{n=0}^{\infty} f^{-n}\left(\mathscr{B}_{\infty}^{k}\right)\right) \cap \mathrm{BB} \quad$ is empty. From the definitions above, it follows that

$$
\begin{aligned}
p_{x}(\varepsilon, S) & =\mu_{1}\left(\left\{y \in I_{x}(\varepsilon) \cap S: L^{+}(x) \neq L^{+}(y)\right\}\right) / \mu_{1}\left(I_{x}(\varepsilon) \cap S\right) \\
& =\sum_{k=1}^{s} \mu_{1}\left(\left\{y \in I_{x}(\varepsilon) \cap \mathscr{B}_{0}^{k}(S): L^{+}(x) \neq L^{+}(y)\right\}\right) / \mu_{1}\left(I_{x}(\varepsilon) \cap S\right) \\
& =\sum_{k=1}^{s} \mu_{1}\left(\left\{y \in I_{x}(\varepsilon) \cap \mathscr{B}_{n}^{k}(S): L^{+}(x) \neq L^{+}(y)\right\}\right) / \mu_{1}\left(I_{x}(\varepsilon) \cap S\right) \\
& =\sum_{k=1}^{s} p_{x}^{k}(\varepsilon, S) \cdot\left(\mu_{1}\left(I_{x}(\varepsilon) \cap \mathscr{B}_{0}^{k}(S)\right) / \mu_{1}\left(I_{x}(\varepsilon) \cap S\right)\right),
\end{aligned}
$$

and furthermore, for each $x \in S$ we have either $p_{x}(\varepsilon, S)=0$ or $p_{x}(\varepsilon, S)=$ $p_{x}^{k}(\varepsilon, S) \cdot\left(\mu_{1}\left(I_{x}(\varepsilon) \cap \mathscr{B}_{0}^{k}(S)\right) / \mu_{1}\left(I_{x}(\varepsilon) \cap S\right)\right)>0$, for some unique integer $k, 1 \leqq k \leqq S$. This implies that

$$
\begin{aligned}
p(\varepsilon, S) & =\int_{S} p_{x}(\varepsilon, S) d x \\
& =\int_{S} \sum_{k=1}^{s} p_{x}^{k}(\varepsilon, S) \cdot\left(\mu_{1}\left(I_{x}(\varepsilon) \cap \mathscr{B}_{0}^{k}(S)\right) / \mu_{1}\left(I_{x}(\varepsilon) \cap S\right)\right) d x \\
& =\sum_{k=1}^{s} \int_{S} p_{x}^{k}(\varepsilon, S) \cdot\left(\mu_{1}\left(I_{x}(\varepsilon) \cap \mathscr{B}_{0}^{k}(S)\right) / \mu_{1}\left(I_{x}(\varepsilon) \cap S\right)\right) d x \\
& =\sum_{k=1}^{s}\left(\mu_{1}\left(I_{x}(\varepsilon) \cap \mathscr{B}_{n}^{k}(S)\right) / \mu_{1}\left(I_{x}(\varepsilon) \cap S\right)\right) \cdot \int_{S \cap \mathscr{B}_{n}^{k}(S)} p_{x}^{k}(\varepsilon, S) d x
\end{aligned}
$$


This together with the combinatorial results in $[\mathrm{Nu} 1, \mathrm{Nu} 2]$ are the main ingredients for adapting the proof of Proposition 4-14 and yielding $\lim _{\varepsilon \rightarrow 0} \frac{\ln p(\varepsilon, S)}{\ln \varepsilon}$ $=1-\max _{1 \leqq k \leqq s} d_{H}\left(\mathscr{B}_{\infty}^{k}(S)\right)$. Hence, $d_{u}(S \cap \mathrm{BB})=d_{H}(S \cap \mathrm{BB})$.

The conclusion is that for $m=1$, the box-counting dimension $d_{b}(S \cap \mathrm{BB})$, the Hausdorff dimension $d_{H}(S \cap \mathrm{BB})$ and the uncertainty dimension $d_{u}(S \cap \mathrm{BB})$ of the intersection of $S$ with the basin boundary BB are all equal.

4-3. Proofs of the Two-Dimensional Results. In this subsection, we prove the theorem for the two dimensional diffeomorphisms. We present in 4-3A some auxiliary results that we use in the proof. The proofs of these results and the proof of the theorem are given in Sect. 4-3B.

4-3A. Two-Dimensional Intermediate Results. In this subsection, we present several auxiliary results that we will use in the proof of the theorem.

Let $\Gamma_{k}(1 \leqq k \leqq v)$ be as in Sect.4-1, that is, each $\Gamma_{k}$ is a basic set in the basin boundary. For each $k$, write $\mathscr{R}_{0}^{k}$ for a block partition of $\Gamma_{k}$. The basic sets in the basin boundary can be partially ordered. We say $\Gamma_{i} \ll \Gamma_{j}$ if the stable set $W^{s}\left(\Gamma_{i}\right)$ intersects the unstable set $W^{u}\left(\Gamma_{j}\right)$.

Lemma 4-15. The basic sets in the basin boundary can be indexed such that for all $i$, $k(1 \leqq i, k \leqq v)$

$$
\Gamma_{i} \ll \Gamma_{k} \quad \text { if } i \leqq k .
$$

The next result says that the dimension of the basin boundary in the block partition $\mathscr{R}_{0}^{k}$ of any basic set $\Gamma_{k}$ in the basin boundary equals the maximum value of the dimension of the basin boundary in the block partitions $\mathscr{R}_{0}^{j}$ of $\Gamma_{j}$ for all $j(k \leqq j \leqq v)$ such that the stable set $W^{s}\left(\Gamma_{k}\right)$ intersects the unstable set $W^{u}\left(\Gamma_{j}\right)$. Here again, "dimension" refers to any of the three concepts of dimension in this paper.

Lemma 4-16. For each integer $k(1 \leqq k \leqq v)$, we have $\operatorname{dim}\left(\mathscr{R}_{0}^{k} \cap \mathrm{BB}\right)$ $\left.=\max _{j} \operatorname{dim}\left(\mathscr{R}_{0}^{j} \cap \mathrm{BB}\right)\right)$, where the maximum is taken over those integers $j$ for which $W^{s}\left(\Gamma_{k}\right)$ intersects $W^{u}\left(\Gamma_{j}\right)$.

The next two results say that the Hausdorff dimension, the box-counting dimension, and the uncertainty dimension of the basin boundary in a block partition of any basic set in the basin boundary are all three equal.

Proposition 4-17. For each integer $k(1 \leqq k \leqq s)$, we have

$$
d_{H}\left(\mathscr{R}_{0}^{k} \cap \mathrm{BB}\right)=d_{b}\left(\mathscr{R}_{0}^{k} \cap \mathrm{BB}\right) .
$$

Proposition 4-18. For each integer $k(1 \leqq k \leqq s)$, we have

$$
d_{H}\left(\mathscr{R}_{0}^{k} \cap \mathrm{BB}\right)=d_{u}\left(\mathscr{R}_{0}^{k} \cap \mathrm{BB}\right)
$$

4-3B. Proofs of the Two-Dimensional (Intermediate) Results. The proof of the Lemmas 4-15 and 4-16 are left to the reader. For every $x \in M$ and for each $\varepsilon>0$, we write $D_{x}(\varepsilon)$ for the open disk in $M$ with radius $\varepsilon$ that is centered at $x$, that is, $D_{x}(\varepsilon)=\left\{y \in \mathbb{R}^{2}:\|x-y\|<\varepsilon\right\}$, which we call $\varepsilon$-disk of $x$. Similarly as in the one dimensional case, we want to introduce a quantity that indicates the relative 
Lebesgue measure of the set of points in any $\varepsilon$-disk $D_{x}(\varepsilon)$. Let $\mu_{2}$ denote the two dimensional Lebesgue measure. For each integer $k(1 \leqq k \leqq v)$, for every $\varepsilon>0$, and each point $x \in M$, we first define

$$
P_{x}^{k}(\varepsilon)=\mu_{2}\left(\left\{y \in D_{x}(\varepsilon) \cap \mathscr{R}_{0}^{k}: L^{+}(x) \neq L^{+}(y)\right\}\right) / \mu_{2}\left(D_{x}(\varepsilon) \cap \mathscr{R}_{0}^{k}\right),
$$

and then

$$
P^{k}(\varepsilon)=\int_{\mathscr{R}_{0}^{k}} P_{x}^{k}(\varepsilon) d x .
$$

The following result compares 1 and 2 dimensional integrals of $P_{x}^{k}(\varepsilon)$.

Lemma 4-19. For each integer $k, 1 \leqq k \leqq v$, there exist $\varepsilon>0$ and constants $C_{m}^{k}>0$ and $C_{M}^{k}>0$ such that

$$
C_{m}^{k} \cdot \int_{\left(I_{k}^{u} \cap \mathscr{R}^{k}\right)} p_{x}^{k}(\varepsilon) d x \leqq P^{k}(\varepsilon) \leqq C_{M}^{k} \cdot \int_{\left(I_{k}^{u} \cap \mathscr{R}^{k}\right)} p_{x}^{k}(\varepsilon) d x
$$

where $\mathscr{R}^{k}=\left\{\mathscr{R}_{n}^{k}\right\}_{n=1}^{N(k)}$ and $I_{k}^{u}$ are as in Proposition 4-2.

Proof. Let integer $k, 1 \leqq k \leqq v$, be given. Let $\mathscr{R}^{k}=\left\{\mathscr{R}_{n}^{k}\right\}_{n=1}^{N(k)}$ and $I_{k}^{u}$ be as in the Proposition. From Sect. 4-1 we know that $\mathscr{R}^{k}$ is contained in an open neighborhood of $\Gamma_{k}$ on which both a stable foliation $\mathscr{F}_{k}^{s}$ and an unstable foliation $\mathscr{F}_{k}^{u}$ exist. We will refer to a component of $\mathscr{F}_{k}^{s} \cap \mathscr{R}^{k}$ (or $\mathscr{F}_{k}^{u} \cap \mathscr{R}^{k}$ ) as a stable segment (unstable segment) of the block partition $\mathscr{R}^{k}$. Let $R$ be any block in $\mathscr{R}^{k}$, and let $I_{R}$ denote the unstable segment $I_{k}^{u} \cap R$. Let US be any unstable segment in $R$. The map $h_{\mathrm{US}}$ : US $\rightarrow I_{R}$ defined by projecting along the leaves of the stable foliation $\mathscr{F}_{k}^{s}$ is a diffeomorphism, and in fact Proposition 4-3 implies that $h_{\mathrm{US}}$ is a $C^{1+\alpha_{-}}$ diffeomorphism, for some $\alpha>0$. Since US may be any unstable segment in $R$ and $R$ is an arbitrarily chosen block of $\mathscr{R}^{k}$, we conclude (by using the fact that $\mathscr{R}^{k}$ is compact) that there exist $\varepsilon>0$ and constants $C_{m}^{k}>0, C_{M}^{k}>0$ such that $C_{m}^{k} \cdot \int_{\left(I_{k}^{u} \cap \mathscr{R}^{k}\right)} p_{x}^{k}(\varepsilon) d x \leqq P^{k}(\varepsilon) \leqq C_{M}^{k} \cdot \int_{\left(I_{k}^{u} \cap \mathscr{R}^{k}\right)} p_{s}^{k}(\varepsilon) d x$.

Remark. One can obtain a somewhat better result than Lemma 4-19. Choosing the coordinates to be the natural coordinates of the block, $P^{k}(\varepsilon)$ is equal to some one-dimensional integral, namely

$$
P^{k}(\varepsilon)=C_{m}^{k} \cdot \int_{\left(I_{k}^{u} \cap \mathscr{R}^{k}\right)} p_{x}^{k}(\varepsilon) d x=C_{M}^{k} \cdot \int_{\left(I_{k}^{u} \cap \mathscr{R}^{k}\right)} p_{x}^{k}(\varepsilon) d x .
$$

Lemma 4-20. For each integer $k, 1 \leqq k \leqq v$, we have

$$
\lim _{\varepsilon \rightarrow 0} \frac{\ln P^{k}(\varepsilon)}{\ln \varepsilon}=1-d_{H}\left(I_{k}^{u} \cap \mathscr{R}^{k} \cap \mathrm{BB}\right)=2-d_{H}\left(\mathscr{R}^{k} \cap \mathrm{BB}\right) .
$$

Proof. Apply Proposition 4-14 and Lemma 4-19.

Proof of Proposition 4-17. Apply Propositions 4-2, 4-3, and 4-8.

Proof of Proposition 4-18. Apply Propositions 4-2, 4-3, and Lemma 4-20.

Proof of the Two Dimensional Theorem. This proof follows immediately from the proof of the one-dimensional theorem, Lemma 4-20, and Propositions 4-17 and 4-18. 


\section{References}

[BP] Berman, A., Plemmons, R.J.: Nonnegative Matrices in the Mathematical Sciences. New York: Academic Press 1979

[B] Bowen, R.: Equilibrium States and the Ergodic Theory of Anosov Diffeomorphisms. Lecture Notes in Mathematics vol. 470. Berlin, Heidelberg, New York: Springer 1975

[E] Eggleston, H.G.: Sets of fractional dimension which occur in some problems of number theory. Proc. Lond. Math. Soc. 54, 42-93 (1951/52)

[FOY] Farmer, D., Ott, E., Yorke, J.A.: The dimension of chaotic attractors. Physica 7D, 153-180 (1983)

[GH] Guckenheimer, J., Holmes, P.: Nonlinear Oscillations, Dynamical Systems, and Bifurcations of Vector Fields. Applied Mathematical Sciences vol. 42. Berlin, Heidelberg, New York: Springer 1983

[GMOY] Grebogi, C., McDonald, S.W., Ott, E., Yorke, J.A.: Final state sensitivity: An obstruction to predictability. Phys. Lett. 99A, 415-418 (1983)

[GNOY] Grebogi, C., Nusse, H.E., Ott, E., Yorke, J.A.: Basic sets: Sets that determine the dimension of basin boundaries. In: Dynamical Systems, Alexander, J.C. (ed.). Proceedings of the University of Maryland 1986-87. Lecture Notes in Math. vol. 1342, pp. 220-250. Berlin, Heidelberg, New York: Springer 1988

[GOY] Grebogi, C., Ott, E., Yorke, J.A.: Basin boundary metamorphoses: Changes in accessible boundary orbits. Physica 24D, 243-262 (1987)

[LY] Lasota, A., Yorke, J.A.: On the existence of invariant measures for piecewise monotonic transformations. Trans. Am. Math. Soc. 186, 481-488 (1973)

[Ma] Mané, R.: Ergodic Theory and Dynamical Systems. (Translated from the Portuguese by S. Levy.) Ergebnisse der Mathemathik und ihrer Grenzgebiete vol. 3. Folge, Band 8. Berlin, Heidelberg, New York: Springer 1987

[MGOY] McDonald, S.W.: Grebogi, C., Ott, E., Yorke, J.A.: Fractal basin boundaries. Physica D 17, 125-153 (1985)

[Mi] Milnor, J.: On the concept of attractor. Commun. Math. Phys. 99, 177-195 (1985). Comments "On the concept of attractor": corrections and remarks. Commun. Math. Phys. 102, 517-519 (1985)

[NP] Newhouse, S., Palis, J.: Hyperbolic nonwandering sets on two-dimensional manifolds. In: Dynamical Systems, Peixoto, M.M. (ed.), pp. 293-301. New York and London: Academic Press 1973

[Nu1] Nusse, H.E.: Asymptotically periodic behaviour in the dynamics of chaotic mappings. SIAM J. Appl. Math. 47, 498-515 (1987)

[Nu2] Nusse, H.E.: Qualitative analysis of the dynamics and stability properties for Axiom $A$ maps. J. Math. Analysis Appl. 136, 74-106 (1988)

[NY1] Nusse, H.E., Yorke, J.A.: A procedure for finding numerical trajectories on chaotic saddles. Physica D 36, 137-156 (1989)

[NY2] Nusse, H.E., Yorke, J.A.: Analysis of a procedure for finding numerical trajectories close to chaotic saddle hyperbolic sets. Ergod. Theory Dyn. Syst. 11, 189-208 (1991)

[PT] Palis, J., Takens, F.: Homoclinic bifurcations and hyperbolic dynamics. $16^{\circ}$ Colóquio Brasileiro Matemática, IMPA, 1987

[P] Pelikan, S.: A dynamical meaning of fractal dimension. Trans. Am. Math. Soc. 292, 695-703 (1985)

[T] Takens, F.: Limit capacity and Hausdorff dimension of dynamically defined Cantor sets. In: Dynamical Systems Valparaiso 1986, Bamón, R., Labarca, R., Palis, J. Jr. (eds.). Proceedings of the Symposium held in Valparaiso, Chile. Lecture Notes in Math. vol. 1331, pp. 196-212. Berlin, Heidelberg, New York: Springer 1988 
